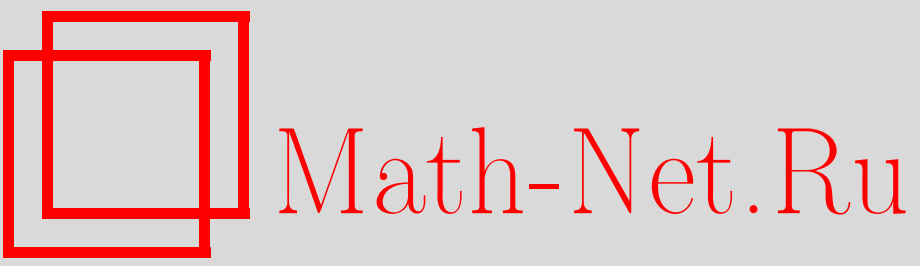

Л. А. Сахнович, Сравнение квантового и классического подходов в статистической физике, ТМФ, 2000, том 123, номер 3, 516-520

DOI: https://doi.org/10.4213/tmf620

Использование Общероссийского математического портала Math-Net.Ru подразумевает, что вы прочитали и согласны с пользовательским соглашением

http://www.mathnet.ru/rus/agreement

Параметры загрузки:

IP: 34.239 .49 .27

26 апреля 2023 г., 13:00:54 
ТЕОРЕТИЧЕСКАЯ

И МАТЕМАТИЧЕСКАЯ

ФИЗИКА

Том 123, № 3

июнь, 2000

(C) 2000 г. $\quad$ Л.А. Сахнович*

\section{СРАВНЕНИЕ КВАНТОВОГО И КЛАССИЧЕСКОГО ПОДХОДОВ В СТАТИСТИЧЕСКОЙ ФИЗИКЕ}

Обсуждаются гипотезы, относящиеся к поведению средней энергии равновесной системы в квантовой теории и средней энергии той же системы в классической теории. Дается обоснование высказанных гипотез для частных случаев осциллятора и потенциальной ямы.

\section{1. ВВЕДЕНИЕ}

Известны результаты, относящиеся к квазиклассическому предельному переходу. Пайерлс рассмотрел свободную энергию

$$
F_{\mathrm{q}}=-T \ln \sum_{n} \exp \left(-\frac{E_{n}(h)}{T}\right)
$$

и нашел основной член ее разложения по степеням $h$. Вигнер, Уленбек и Гроппер определили следующий член этого разложения $[1, \S 32,33]$.

Данная статья посвящена не предельным переходам, а сравнению по величине квантовых и классических результатов для случая равновесной системы.

Через $\bar{E}_{\mathrm{q}}(T, h)$ обозначим среднюю энергию равновесной системы в квантовой теории, а через $\bar{E}_{\mathrm{c}}(T)$ - среднюю энергию той же системы в классической теории $(T-$ температура). Через $S_{\mathrm{q}}(T, h)$ обозначим энтропию равновесной системы в квантовой теории.

В статье обсуждаются следующие гипотезы.

ГиПотеЗА 1. Для равновесных систем справедливо неравенство

$$
\bar{E}_{\mathrm{q}}(T, h)>\bar{E}_{\mathrm{c}}(T) .
$$

ГИПоТЕЗА 2. Средняя әнергия равновесной системы в квантовой теории $\bar{E}_{\mathrm{q}}(T, h)$ монотонно возрастает по переменной $h$.

\footnotetext{
* Украинская государственная академия связи им. А. С. Попова, Одесса, Украина
} 
ГиПотеЗА 3. Энтропия $S_{\mathrm{q}}(T, h)$ равновесной системы монотонно убывает по переменной $h$.

Справедливость сформулированных гипотез подтверждается в случае простейших примеров (гармонический осциллятор, потенциальная яма).

Заметим, что сравнение результатов квантового и классического подходов представляет большой интерес как с прикладной, так и с методологической точек зрения.

\section{2. ОСНОВНЫЕ ОПРЕДЕЛЕНИЯ}

Рассмотрим систему, которая может находиться в одном из $N$ состояний $(1 \leqslant N<$ $\infty)$. Через $P_{n}$ обозначим вероятность того, что система находится в состоянии с номером $n(1 \leqslant n \leqslant N)$, а через $E_{n}$ - соответствуюшую энергию. Средняя энергия $\bar{E}_{\mathrm{q}}(T, h)$ в каноническом ансамбле Гиббса определяется формулой

$$
\bar{E}_{\mathrm{q}}(T, h)=\sum_{n=1}^{N} E_{n}(h) P_{n}(T, h),
$$

где

$$
\begin{aligned}
& P_{n}(T, h)=\exp \left[-\frac{E_{n}(h)}{T}\right] \Phi_{\mathrm{q}}^{-1}(T, h), \\
& \Phi_{\mathrm{q}}(T, h)=\sum_{n=1}^{N} \exp \left[-\frac{E_{n}(h)}{T}\right] .
\end{aligned}
$$

Пусть энергия системы $E(p, q)$ является функцией координат $q$ и импульсов $p$. Средняя энергия системы в фазовом пространстве имеет вид

$$
\bar{E}_{\mathrm{c}}(T)=\iint E(p, q) W(p, q) d p d q,
$$

где согласно формуле Гиббса

$$
W(p, q)=\exp \left[-\frac{E(p, q)}{T}\right] \Phi_{c}^{-1}(T)
$$

и

$$
\Phi_{\mathrm{q}}(T)=\iint \exp \left[-\frac{E(p, q)}{T}\right] d p d q
$$

\section{3. ГАРМОНИЧЕСКИЙ ОСЦИЛЛЯТОР}

В случае гармонического осциллятора, как известно [2],

$$
E_{n}=h \omega\left(n-\frac{1}{2}\right), \quad n=1,2, \ldots,
$$


т.е. в этом случае

$$
\Phi_{\mathrm{q}}(T, h)=\sum_{n=1}^{\infty} \exp \left[-\frac{h \omega\left(n-\frac{1}{2}\right)}{T}\right]=\exp \left[-\frac{h \omega}{2 T}\right]\left[1-\exp \left(-\frac{h \omega}{T}\right)\right]^{-1}
$$

Пользуясь формулой

$$
\sum_{n=1}^{\infty} e^{-\alpha n} n=\frac{e^{-\alpha}}{\left(1-e^{-\alpha}\right)^{2}}
$$

получаем, что

$$
\bar{E}_{\mathrm{q}}(T, h)=\sum_{n=1}^{\infty} \exp \left[-\frac{h \omega\left(n-\frac{1}{2}\right)}{T}\right] h \omega\left(n-\frac{1}{2}\right) \Phi_{\mathrm{q}}^{-1}(T, h)=\frac{h \omega}{2 \operatorname{th} \frac{h \omega}{2 T}} .
$$

Из последней формулы следует, что

$$
\frac{\partial \bar{E}_{\mathrm{q}}(T, h)}{\partial h}=\frac{\omega}{2}\left[\frac{\exp \frac{h \omega}{T}-\exp \left(-\frac{h \omega}{T}\right)}{4}-\frac{h \omega}{T}\right] \operatorname{sh}^{-2} \frac{h \omega}{2 T}>0
$$

т.е. функция $\bar{E}_{\mathrm{q}}(T, h)$ монотонно возрастает по переменной $h$ и в этом случае гипотеза 2 подтверждается.

Из этого факта и соотношения

$$
\lim _{h \rightarrow 0} \bar{E}_{\mathrm{q}}(T, h)=T
$$

следует, что

$$
\bar{E}_{\mathrm{q}}(T, h)>T=\bar{E}_{\mathrm{c}}(T) .
$$

Таким образом, в случае гармонического осциллятора гипотеза 1 также подтверждается.

\section{4. ПОТЕНЦИАЛЬНАЯ ЯМА}

В случае потенциальной ямы спектр $E_{n}$ системы совпадает со спектром краевой задачи

$$
\Psi^{\prime \prime}(x)+\frac{2 m}{h^{2}} E \Psi=0, \quad \Psi(0)=\Psi(a)=0,
$$

т.е. справедливо равенство

$$
E_{n}(h)=\frac{h^{2} \pi^{2}}{2 m a^{2}} n^{2}, \quad n=1,2,3, \ldots
$$

Используя формулы (1)-(3), имеем

$$
\bar{E}_{\mathrm{q}}(T)=\frac{\pi^{2} h^{2}}{2 m a^{2}} \sum_{n=1}^{\infty} n^{2} e^{-\frac{n^{2}}{\lambda}}\left[\sum_{n=1}^{\infty} e^{-\frac{n^{2}}{\lambda}}\right]^{-1}
$$


где

$$
\lambda=\frac{2 m a^{2} T}{\pi^{2} \hbar^{2}}
$$

Посчитаем теперь величину $\bar{E}_{\mathrm{c}}(T)$. В случае потенциальной ямы имеем

$$
E(p, q)= \begin{cases}\frac{1}{2 m} p^{2}, & 0<q<a \\ \infty, & q \notin[0, a]\end{cases}
$$

Из формул (4)-(6) следует, что

$$
\bar{E}_{\mathrm{c}}(T)=\frac{1}{2 m} \int_{-\infty}^{\infty} p^{2} e^{-\frac{p^{2}}{2 m T}} d p\left[\int_{-\infty}^{\infty} e^{-\frac{p^{2}}{2 m T}} d p\right]^{-1}=\frac{T}{2}
$$

Строго доказать гипотезы 1 и 2 даже в рассматриваемом простом случае нам не удалось (гипотезу 3 мы рассмотрим далее отдельно). Поэтому мы прибегли к подсчетам на компьютере. При этом мы считали, что выполнено нормировочное условие

$$
\frac{\pi h^{2}}{2 m a^{2}}=1
$$

В широком диапазоне температур $(0.1 \leqslant T \leqslant 1000)$ гипотеза 1 подтверждается.

Заметим, что в ряде задач величину $\bar{E}_{\mathrm{c}}(T)$ проще подсчитать, чем $\bar{E}_{\mathrm{q}}(T)$. Зная величину $\bar{E}_{\mathrm{c}}(T)$, мы согласно гипотезе 1 получаем оценку для $\bar{E}_{\mathrm{q}}(T)$. Разность $\Delta=\bar{E}_{\mathrm{q}}(T)-$ $\bar{E}_{\mathrm{c}}(T)$ может служить мерой квантового эффекта.

\section{5. ЗАВИСИМОСТЬ ЭНТРОПИИ ОТ $h$}

Справедливость гипотезы 3 , относяшейся к энтропии системы

$$
S_{\mathrm{q}}(T, h)=-\sum_{n} P_{n} \ln P_{n}
$$

можно доказать для широкого класса задач. Для этого запишем формулу (9) в виде

$$
S_{\mathrm{q}}(T, h)=\frac{\bar{E}_{\mathrm{q}}(T, h)}{T}+\ln \Phi_{\mathrm{q}}(T, h)
$$

УТВЕРЖДЕНИЕ. Пусть выполнено соотношение

$$
E_{n}(h)=\varphi(h) E_{n}, \quad \varphi(h)>0
$$

где $E_{n}$ не зависит от $h$, а $\varphi(h)$ монотонно возрастает. Тогда соответствующая функиия $S_{\mathrm{q}}(T, h)$ монотонно убивает по переменной $h$. 
ДокАЗАТЕльСтво. В случае (11) формулу (10) можно записать в виде

$$
S_{\mathrm{q}}(T, h)=\lambda \sum_{n} E_{n} e^{-\lambda E_{n}}\left[\sum_{n} e^{-\lambda E_{n}}\right]^{-1}+\ln \sum_{n} e^{-\lambda E_{n}}=\Psi(\lambda),
$$

где $\lambda=\varphi(h) / T$. Вследствие формулы (12) верно соотношение

$$
\Psi^{\prime}(\lambda)=\lambda\left[-\sum_{n} E_{n}^{2} e^{-\lambda E_{n}} \sum_{m} e^{-\lambda E_{m}}+\left(\sum_{n} E_{n} e^{-\lambda E_{m}}\right)^{2}\right] \Phi^{-2}(\lambda),
$$

где

$$
\Phi(\lambda)=\sum_{n} e^{-\lambda E_{n}}
$$

Равенство (13) может быть записано в виде

$$
\begin{aligned}
\Psi^{\prime}(\lambda)= & \lambda\left[-\sum_{n} E_{n}^{2} e^{-2 \lambda E_{n}}-\sum_{n \neq m}\left(E_{n}^{2}+E_{m}^{2}\right) e^{-\lambda\left(E_{n}+E_{m}\right)}+\right. \\
& \left.+\sum_{n} E_{n}^{2} e^{-2 \lambda E_{n}}+2 \sum_{n \neq m} E_{n} E_{m} e^{-\lambda\left(E_{n}+E_{m}\right)}\right] \Phi^{-2}(\lambda),
\end{aligned}
$$

т.е.

$$
\Psi^{\prime}(\lambda)=-\lambda \sum_{n \neq m}\left(E_{n}-E_{m}\right)^{2} e^{-\lambda\left(E_{n}+E_{m}\right)} \Phi^{-2}(\lambda)<0,
$$

откуда следует справедливость доказываемого утверждения.

СлеДСТВИЕ. Гипотеза 3 верна для гармонического осииллятора, потенциальной ямы и для многомерной потенииальной ямы.

В самом деле, по формуле (7) для гармонического осциллятора $\varphi(h)=h$, т.е. выполняется условие (11). По формуле (8) для одномерной потенциальной ямы

$$
\varphi(h)=h^{2},
$$

т.е. условие (11) тоже выполнено.

Рассмотрим теперь в многомерной области $D$ с гранищей $\Gamma_{D}$ спектральную задачу

$$
-h^{2} \Delta u=E_{n}(h) u,\left.\quad u\right|_{\Gamma}=0,
$$

которая описывает многомерную потенциальную яму. Легко видеть, что для этого класса задач справедливо равенство (14), т.е. выполняется условие (11).

\section{Список литературы}

[1] Л. Д. Ландау, Е. М. Лифииц. Статистическая физика. М.: Наука, 1964.

[2] Л. Д. Ландау, Е. М. Лифииц. Квантовая механика, нерелятивистская теория. М.: Физматгиз, 1963.

Поступила в редакцию 1.III.1999 г., после доработки 22.XI.1999 г. 